\title{
Utility and applications of synoptic reporting in pathology
}

This article was published in the following Dove Press journal:

Open Access Bioinformatics

5 August 2010

Number of times this article has been viewed

Waqas Amin'

$S$ Joseph Sirintrapun ${ }^{3}$

Anil V Parwani ${ }^{1,2}$

'Department of Biomedical Informatics, ${ }^{2}$ Department of Pathology

University of Pittsburgh, Pittsburgh, PA, USA; ${ }^{3}$ Department of Pathology, Emory University, Atlanta, GA, USA
Correspondence: Anil V Parwani Shadyside Hospital, UPMC, 5230 Centre Ave, Suite WG02. I0, Pittsburgh, PA 15232, USA

$\mathrm{Tel}+|4| 2623$ I326

Fax + I 4I26826450

Email parwaniav@upmc.edu
Background: Synoptic reports in routine pathology practice provide composite documents that include information from morphology and molecular technologies. It is clear and accurate structured information and developed by incorporating standardized data elements in the form of checklist for pathology reporting. This facilitates pathologists to document their findings and ultimately improve the overall quality of pathology reports.

Objectives: The goal of this review article is to discuss (1) the importance of synoptic reporting in pathology, (2) utility and applications, (3) its impact on pathology reporting and patient care, and (4) the challenges and barriers of implementing synoptic reporting. Pertinent literature will also be reviewed.

Design: The synoptic reporting system provides a complete set of data elements in the form of synoptic templates or "worksheets" for pathology tumor reporting based on the World Health Organization (WHO) Classification and the College of American Pathologists (CAP) Cancer Checklists. These standards provide most updated and supplemented classification scheme, specimen details, and staging as well as prognostic information. Data from synoptic reporting tool can be imported to a relational database where they are organized and efficiently searched and retrieved. Since search and retrieval are streamlined, synoptic databases enhance basic science, clinical, and translational cancer research.

Conclusion: Synoptic reporting facilitates a standard based structured method for entering the diagnostic and prognostic information in accurate and consistent fashion for a particular pathology specimen, thus reducing transcription services, specimen turnaround time, and typographical and transcription errors. The structured data can be imported into the Laboratory Information Service (LIS) database, which facilitates swift data access and improved communication for cancer management. Finally, these synoptic templates act as a robust medium of high-quality data from the various biospecimens, which can be shared across multiple on-going research projects to enhance basic and translational research.

Keywords: synoptic reporting, pathology

\section{Introduction}

Cancer diagnoses make up a majority of specimens reviewed in pathology labs. Contemporary surgical pathology reports have traditionally provided basic information such as tumor type, grade, margin involvement, and angiolymphatic invasion, all of which give a morphologic perspective on general tumor behavior but little about patient-specific prognostic behavior. Clinicians demand more information to evaluate and manage their individual patients, ie, prognostic information such as hormone receptor status or oncogene expression, which can determine a patient's response to adjuvant therapy. With our understanding of tumor biology ever expanding along 
with more molecular and translational features defined, the surgical pathology report has become a prime source for conveying not only the morphologist's perspective on the tumor but also information generated by these new molecular and translational technologies. The modern surgical pathology report has evolved to become complex and provide detailed information on tumour biology that encompasses both information from morphology as well as these new molecular and translational technologies. ${ }^{1}$

Because of its importance, the surgical pathology report must be clear, accurate, and thorough. Unfortunately, traditional narrative and descriptive pathology reports, although reflective of a given pathologist's style, show significant variability in format, context, and content. With the increasing complexity demanded of the modern surgical pathology report, necessary elements are occasionally omitted. ${ }^{1}$ Zarbo et al studied 15,940 pathology reports of colorectal cancer and reported that basic crucial elements such as gross tumor size, depth of tumor invasion, resection margins, and tumor grades were often absent. ${ }^{2}$

In addressing this issue in 1993, Rosai proposed standardized reporting of surgical pathology diagnoses for the major tumors. In 2002, the American College of Surgeons Commission on Cancer (ACOS COC) reiterated this mandate by recommending mandatory cancer protocols. Considerable work by morphologists, researchers, and informaticians was performed to develop mechanisms that ensured quality and uniformity among pathology reports regardless of the institution of origin; hence the College of American Pathologists (CAP) Cancer Protocols and Checklists was produced. ${ }^{1,3-9}$

Checklists or synoptic reporting, as in the CAP Cancer protocols, provides a structured and pre-formatted method for entering clinically and morphologically relevant details of surgical specimens. A checklist (synoptic) format makes reporting efficient, uniform, and complete, especially for the major tumors.

Ideally synoptic reporting enters information as discrete data elements. With the CAP checklists, the data elements lists are derived from established scientifically proven data. In addition, some discrete elements are "flagged" to be used to validate future changes in the existing checklist, allowing for evaluation and improvement with quality assurance. Ideally with synoptic reporting, discrete data elements are passed to a relational database where they are organized and efficiently searched and retrieved. In contrast, are databases that are based on natural language processes, where there is no logical organization of the words within the report and where search and retrieval of natural word elements are cumbersome. Synoptic reporting makes data search and retrieval streamlined and enhances basic science, clinical, and translational cancer research. ${ }^{3,4,6-9}$

Synoptics provide an "online diagnosis worksheet" that is easily learned and deployed. This encourages pathologists to enter diagnostic information by themselves, obviating the need for transcription services and thereby reducing specimen turnaround time. Communication between the pathologist and clinician is enhanced since synoptic reporting prioritizes the presentation of large amounts of diagnostic information. This is especially relevant for large surgical resections, which can often yield overwhelming amounts of information for clinicians. $^{3,4,6-9}$

In 2003, the University of Pittsburgh Medical Center (UPMC), a huge conglomerate of multiple hospitals and medical centers, along with the Pathology Informatics team proactively initiated the use of digital synoptic worksheets based on CAP protocols to standardize reports for all the UPMC participating hospitals. By January 1, 2004, the American College of Surgeons Commission on Cancer (ACOS COC), which accredits over 1400 cancer treatment centers, ${ }^{10}$ followed suit by requiring participating pathologists to include all scientifically validated data elements from the CAP checklists in their reports for each site and specimen. We describe our experience with Digital Synoptic Worksheet entry from its inception and incorporation into the daily anatomic pathology workflow for the major tumors.

\section{Methods \\ Technology}

The synoptic reporting functions examined in this study were developed at UPMC using the CERNER's CoPathPLUS synoptic reporting module. This work is partially supported by CAP Foundation Rippey Grant for Quality Assurance. The module is fully integrated into our existing laboratory information system, coPathPlus (v2.5.1.83). ${ }^{11}$ The LIS provides a Windows-based user interface organized into workflowrelated "activities", and is built on a relational database platform (Sybase). We modified the CAP checklists into synoptic worksheets for selected organ systems and malignancies. These worksheets also include diagnostic and research information specific to each of UPMC's Centers. The digital synoptic reporting module uses these predefined worksheets to generate a report that contains all the information desired by the clinicians, and all elements considered essential by the pathologists to the pathology report (Figure 1).

The synoptic reporting system consists of four discrete components (Figure 2). The first one is synoptic reporting 


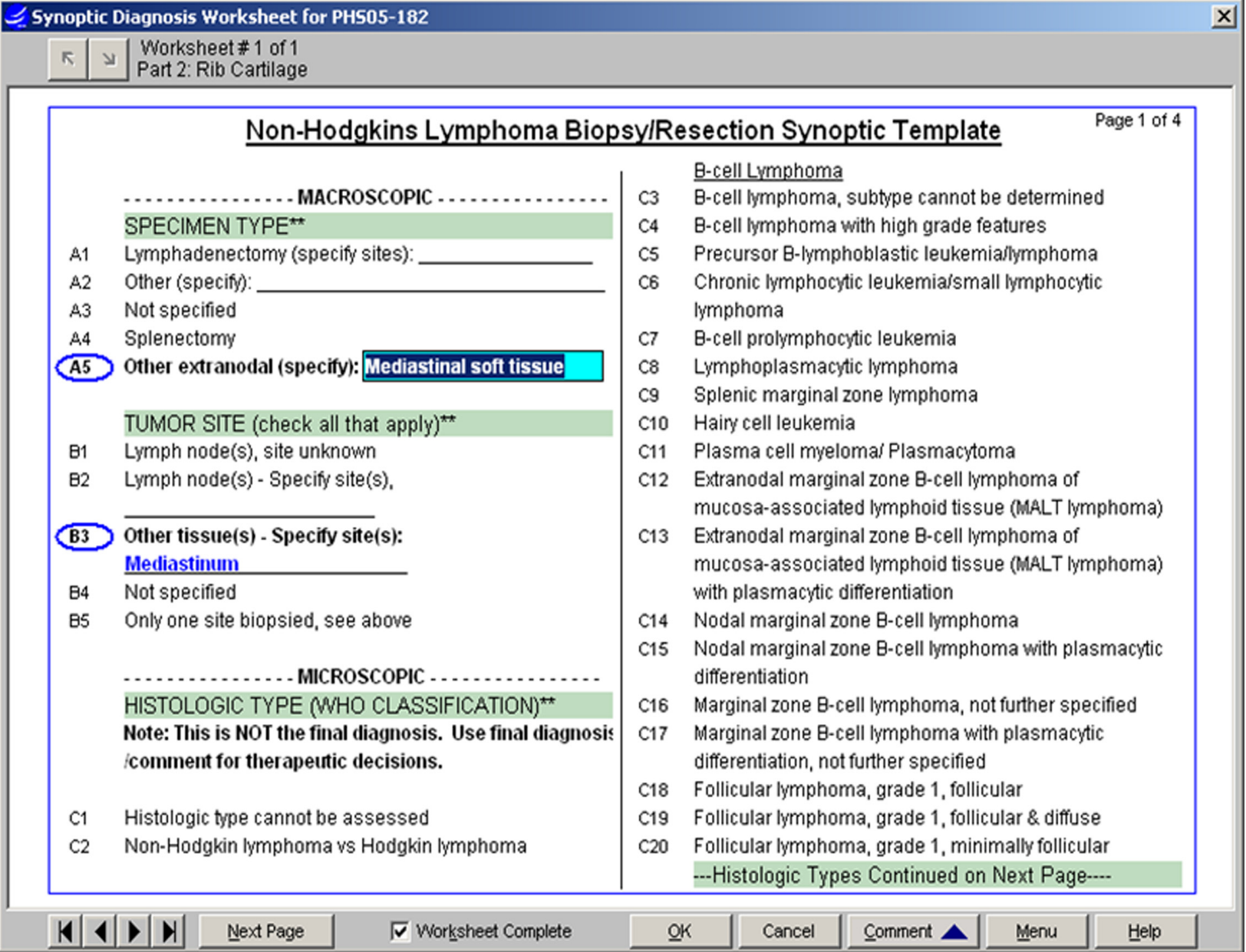

Figure I Presents synoptic reporting: primary entry. Within this interface, the pathologist can enter data electronically. Selections are circled in blue. Using validation logic, the interface ensures that all the necessary elements are completed before a report is generated.

dictionaries which are further subdivided into synoptic sub-dictionaries (Figure 3).

The Categorical dictionary is defined and connected with logical headers in the checklist to which synoptic values are then allocated, for example, histologic type, extent of tumor or tissue type. This dictionary is then used to add particular groups of values to a worksheet and provide a default header for the groups, and may also be used later to facilitate queries. Value dictionary provides a synoptic value

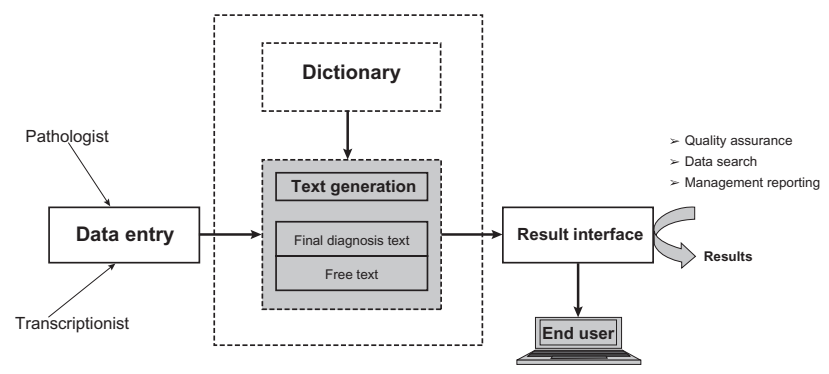

Figure 2 Presents the four components of synoptic reporting tool. entry that is formulated for each particular item that should be on a synoptic worksheet. The values can be selected to include a fill-in text or numeric type field in the dictionary. In order to facilitate query interface or exporting synoptic results systematized nomenclature of medicine-clinical terms (SNOMED-CT) codes may also be allied with the values in this dictionary, to maintain other database later on. The Worksheet dictionary provides synoptic "worksheets" model that are distinct and grouped in this dictionary. The important values on a worksheet are explained in the preceding dictionaries. The grouped values inside the dictionary are placed to require a selection within the group that allows single or multiple selections within the group and leave out printing of the category header in the generated text in which no value is selected. The text character is produced for reporting the group headers, and values can also be selected in this dictionary. The Worksheet group dictionary is linked worksheet "groups", for data query function, which is optional within the synoptic system. The Part type dictionary 


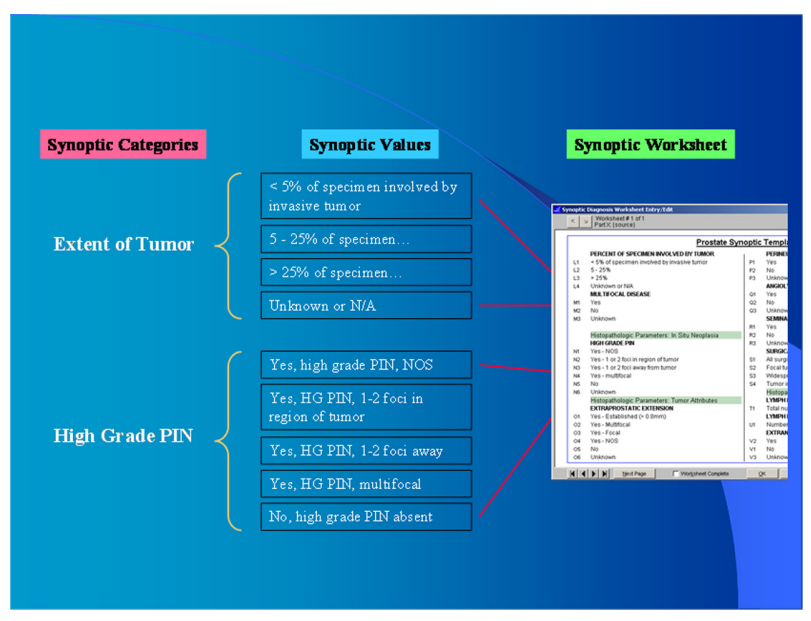

Figure 3 Presents synoptic dictionary structure. Dictionaries provide the foundation by which data are captured as discrete elements. Synoptic values are the most granular elements and these are grouped into synoptic categories. The worksheets reflect this hierarchy. All captured data are stored in a relational database.

permits default synoptic worksheets that are specific to each part type that is assigned to a specimen. ${ }^{12}$

The second component is Specimen data entry and text generation. In the Final Diagnosis Entry/Edit and electronic Sign-out activities; the windows can be painted to enable synoptic data entry/editing in line with editing of other diagnostic text in the report. The pathologist or training staff can directly access and complete the worksheets or the pathologist may dictate the report and the worksheet is completed by a transcriptionist. In case required group values is left vacant or a selected value is filled in with inconsistent formatting, a system warning is issued to the staff generated by Validation logic based on definition of worksheets. This ensures a comprehensive and accurate completion of the worksheet. At the completion of worksheet online, the automated diagnosis text is produced based on specifications in the Synoptic Worksheet definition and intended for the generation of the Final Diagnosis Text field or to a separate text field. Once the system has generated this text, no further changes can be made without corresponding changes to the synoptic values that were the source of that text. This measure serves to protect the text from modification through word processor. Provision of free text comment has been made in the text field separate from the protected text. ${ }^{12}$

The third component is the Results interface, which is an HL-7 interface. This interface can be constructed on an elective basis to send out distinct synoptic data elements via " $\mathrm{Z}$ segment" annex, along with the text-based HL-7 results. ${ }^{12}$

The fourth component is Data search and management reporting, which is a data search capability that is provided via the "Infomaker wizard" tool. This tool facilitates detailed searches of specific specimen and patient parameters in combination with discrete synoptic data fields. There are also several management reports designed to specify the cases with incomplete worksheets and to analyze the implementation of synoptic worksheets by individual pathologists and the type of cases entered, and for searching cases by natural language or SNOMED coding to determine usage of worksheets. ${ }^{12}$

\section{Results}

Synoptic reports have hierarchical construction with dictionaries providing the foundation for each synoptic worksheet. With primary entry, categories have their own defined vocabulary. Categorical vocabulary would include specimen type, histologic type, or extent of tumor. Following that, each category is further subclassified into values. Thus under the category of specimen type, the values dictionary would include "radical prostatectomy", "simple prostatectomy", or "transurethral prostatectomy". With each term defined by dictionaries, the discrete data elements captured in a worksheet can be parsed and organized in a logical order and structure. The data elements that are captured from the synoptic reports are organized in the relational database, thus making the data more amenable to efficient search and retrieval. Users can simply search for cases that have certain value points populated such as radical prostatectomy or "clear cell renal cell carcinoma". This provides a powerful advance over free-text reports, which are seen in the majority of institutions with electronically generated reports. As opposed to synoptic reporting, wording stored from free-text reporting lacks relational structure and thus searches and retrieval processing is notoriously inefficient and slow.

The synoptic reporting module is fully integrated in the anatomic pathology laboratory information service (APLIS), and the worksheets are easily incorporated into the daily sign-out activities without having to go to a separate program or website. During specimen accessioning, worksheets are attached to the case by grossing staff as part of routine grossing protocols. The resident or pathologist would then dictate or select data elements from the worksheet using the online module to create a final diagnosis. If the report is dictated, then a transcriptionist enters the values using the online module. The sign-out pathologist performs final review, discrete data elements from the report are captured and stored on the relational database, and a final report is generated for clinical use (Figure 4a, 4b).

Quality assurance is routinely performed electronically within the framework of the module. Synoptic templates are 


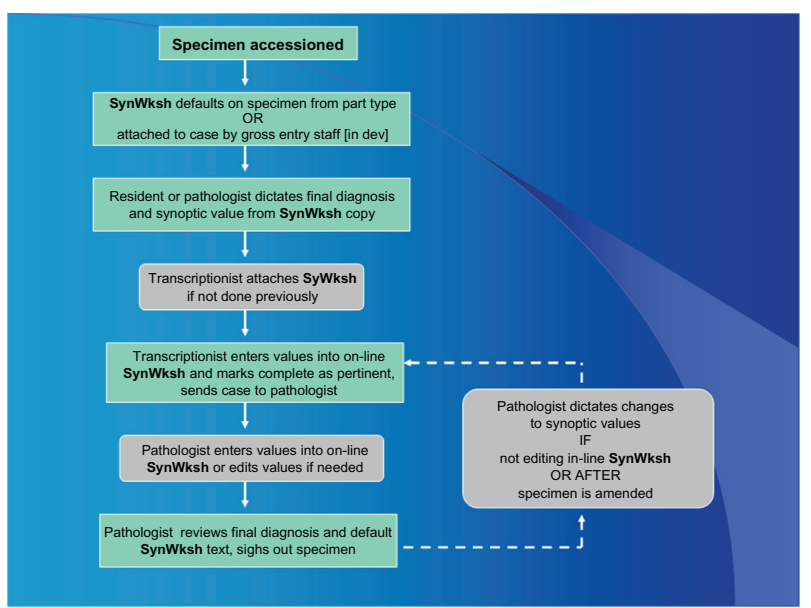

Figure 4a Presents synoptic reporting: workflow. Data can be entered electronically or done by hand. The worksheets are integrated with the APLIS and the workflow, so that reviews and edits are made seamlessly before the report is signed out.

revised, reviewed, and approved for implementation through improvement assessments with the web-based Synoptic Evaluation Application (SEA), introduced in 2004.

Since the deployment of synoptics, a total of 15,166 specimens in our hospital network had synoptic reports completed
(Figure 2-1). The breast/gynecology (7912), genitourinary (4578), gastrointestinal (3583), and lung (1550) were the most commonly utilized synoptics. The use of synoptics has increased greatly each year from 2003-2009 (Figure 5). Interestingly, two subspecialties, genitourinary with prostate biopsies and dermatopathology with melanomas, have complied consistently with digital synoptic usage since its inception in 2003. By 2005, all anatomic pathology subspecialties had begun incorporating digital synoptic tools in their reports. Even rarer malignancies including parathyroid tumors, primary penile tumors, gastrointestinal and Hodgkin lymphomas, and adrenal cortical carcinomas had used their associated corresponding synoptic templates.

\section{Discussion}

Since the implementation of CAP checklists, there is a paucity of information describing the advantages regarding the use of the checklists and their impact on reporting pathology data to the health care team, cancer registry, quality improvement departments, marketing, public health agencies, and research databases. A major reason for this is that most pathology APLISs are not able to support discrete

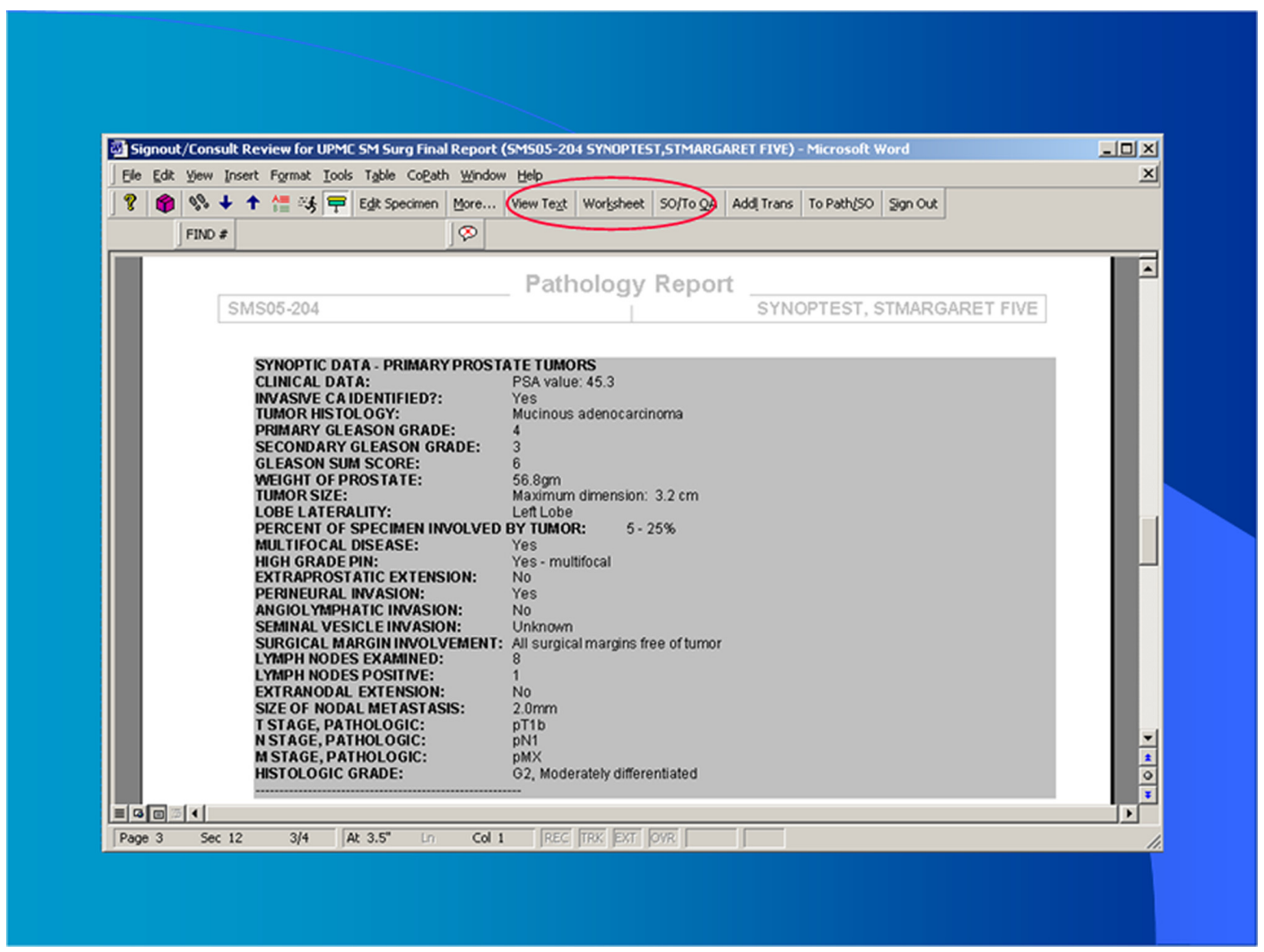

Figure 4b Presents synoptic reporting: report generation and output. After completion of the synoptic worksheet, text descriptors for all selected items are generated and printed in the report. Virtually, each element listed here is stored within the relational database. 


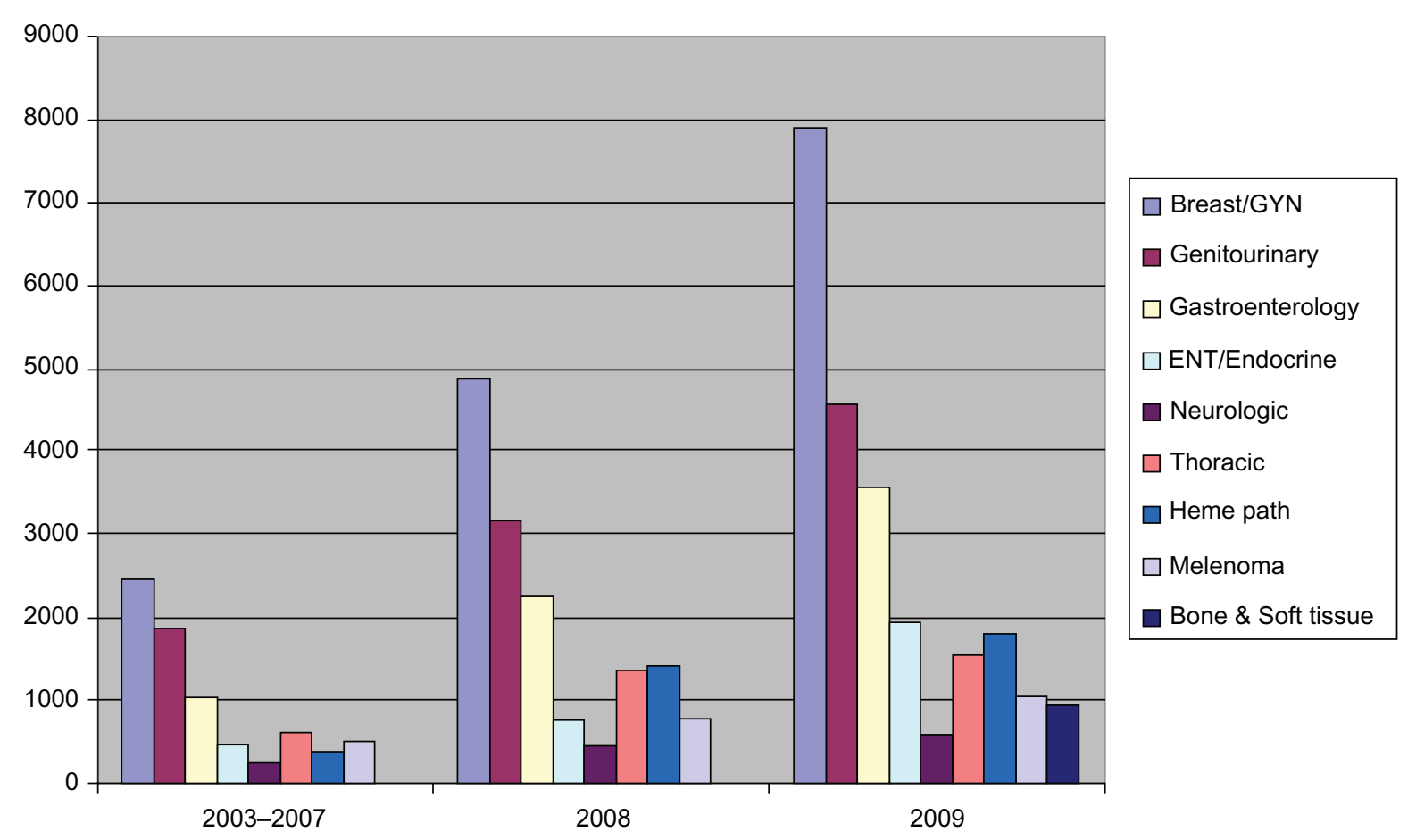

Figure 5 Presents the distribution of synoptic use by organ systems and year.

data capture for synoptic data elements. Therefore, the CAP checklists, despite their initial visionary intent, are often captured as unstructured text blocks within pathology reports at most institutions that use electronic sign-out. Hence the results are databases with cumbersome search, access, and retrieval formats and are of little benefit.

Our intention is to describe the UPMC experience with true digital synoptic reporting utilized at its full potential with discrete data element capture and relational database storage and retrieval. Since 2003, UPMC has used digital synoptic reporting, which has been fully integrated into the existing laboratory information system (LIS), CoPathPlus, from Cerner DHT. The synoptic reporting at UPMC is constantly updated and checked for quality assurance, with all current versions of the CAP checklists incorporated into these synoptic worksheets.

Our analysis showed that synoptic reporting tools employed at UPMC are useful by providing the pathologist with effective worksheets to capture the American College of Surgeons Commission on Cancer required elements based on CAP checklists and protocols and for the purpose of introducing more standardized pathology reports. In all of the worksheets, it was noteworthy that the UPMC worksheets exceeded the American College of Surgeons required CAP elements providing additional data elements which were deemed necessary by representatives of the
UPMC Centers of Excellence and pathologists for their routine practice. The hope would be that these additional elements will play a role in the future to further discoveries in diagnostics and therapeutics related to these neoplastic diseases.

Synoptic reporting provides uniform and standardized data elements through checklists that enable pathologists to make notes of pathological findings in the report by avoiding a free text component. ${ }^{6-9,11}$ Furthermore the use of synoptic reports data entry method generates consistent and standardized reports that optimize the pathology reporting standards with competence for quality assurance and control. An accurate and consistent diagnosis and staging information dictated by pathologists facilitates the clinicians to provide a basis for treatment recommendations and ultimate survival predictions. The checklist item in a synoptic report provides clear and consistent pathological diagnostic information thus reducing the necessity to re-review slides, reducing time spent on signing out. It also improves the assessment of quality of care studies, marketing, and research activities. The cancer registry can also get the benefit from synoptic reporting system by using the synoptic template to pull out common data elements from a completed pathology report to fill the registry environment with subsequent association to the centralized integrated data annotation and query engine for research and data sharing. 
Although the synoptic tool is a novel and interesting way of conveying diagnostic and prognostic information to clinicians, its use is controversial among pathologists. Based on our experience at our institute we have encountered a range of responses when the synoptic tool was introduced. Some pathologists really liked the concept while others vehemently resisted the use of the tool. We did notice that after the tool was deployed for some time and pathologists became familiar with its features and the degree of resistance towards the tool decreased. Various factors play a role in why synoptic tools are not easily accepted by pathologists. Some pathologists were fearful that synoptics are relatively cumbersome and time-consuming because they may require additional steps to enter and/or edit worksheets compared to usual free text reports. This emphasizes the importance of synoptic incorporation within the LIS and workflow as seen here at UPMC. Additionally, critics may feel that synoptics may allow for less flexibility for nuanced diagnoses or microscopic findings. In other words, there may be loss of context within reports. This may be potentially true for rare, controversial, and/or academically interesting tumors. However, for the vast majority of common malignancies, most subtle nuances have widespread acknowledgment such that an individually styled report is not necessary to cover the scope of information needed to be conveyed to both the patient and clinician. Furthermore, in addressing these concerns and to achieve successful synoptic worksheet implementation, there should be collaboration among the subspecialty pathologists and clinicians of each Centers of Excellence in constructing each synoptic worksheet. This normalizes the lines of communication and addresses both the clinical needs and capture of information present and future. Admittedly in our experience, this was not an easy undertaking but one that took many years to develop. Eventually consistency in the use of synoptic reporting will depend on leadership commitment, pathologist's experience, quality of training, and acceptance to this novel and intuitive tool at your institution.

\section{Conclusions}

Clinicians rely on accurate diagnosis and staging information from surgical pathology reports for treatment recommendations and prognostic predictions. Synoptic reports generate consistent and structured data elements, and when placed in a relational APLIS database, there is enabling of quick access to desired diagnostic and prognostic information with improved communication for appropriate therapeutic protocols. From a practical standpoint, synoptics obviate the need for transcription services and reduces specimen turnaround time. Since data elements are consistent typographical and transcription errors are minimized. Being based on the CAP protocols and checklists, data captured from the UPMC synoptics no matter how immense, can be reported in a fair, consistent, and prioritized manner with less concern for omission for critical data elements. With cancer patients now diagnosed and treated in multiple settings, this uniform documentation of communication among health care facilities is demanded. At UPMC, our synoptics closely reflect the CAP checklists and that for the most part UPMC synoptic templates correspond to narrative reports. We have also demonstrated that the synoptic templates have become increasing widespread in their use at the multiple centers at UPMC.

From an academic standpoint, with synoptic reporting both clinical and research relevant data elements are captured. Such uniformity of data capture lends itself to subsequent ease of data viewing and extraction with rapid production of standardized, high-quality data. With more powerful capture of information, key data elements stored in the LIS relational database can be quickly accessed to provide the desired information for research as well as personalized cancer management. Because of this feature, synoptics are being recognized as the future of pathology reports. The Cancer Biomedical Informatics Grid (caBIG), a network of individuals and institutions developing the future infrastructure of cancer biomedical informatics research, has proposed that the CAP cancer checklists for which the UPMC synoptics are based, be the electronic data standard in pathology. ${ }^{13}$ Our study shows that our mission to provide synoptic templates that serve as a conduit for capturing and storing data in a virtual biorepository for translational research and clinically relevant information has been a success.

\section{Acknowledgments}

The authors thank the department of Pathology at the University of Pittsburgh School of Medicine, Pittsburgh, PA, Department of Biomedical Informatics (Anthony L Piccoli and Lisa J Devine) and the Cerner DHT CoPathPlus System.

\section{Disclosure}

No conflicts of interest were declared in relation to this paper.

\section{References}

1. Rosai J. Standardized reporting of surgical pathology diagnoses for the major tumor types. A proposal. The Department of Pathology, Memorial Sloan-Kettering Cancer Center. Am J Clin Pathol. 1993;100(3):240-255.

2. Zarbo RJ, Hoffman GG, Howanitz PJ. Interinstitutional comparison of frozen-section consultation. A College of American Pathologists Q-Probe study of 79,647 consultations in 297 North American institutions. Arch Pathol Lab Med. 1991;115(12):1187-1194. 
3. de Buys Roessingh AS, Laurini RN, Meyrat BJ. Nephrogenic adenoma of the urethra: an unusual cause of hematuria in the child. J Pediatr Surg. 2003;38(8):E8-E9.

4. Kibar Y, Sumer F, Yildirim I, Gamsizkan M, Avci A, Dayanc M. Nephrogenic adenoma of the bladder in a girl with Turner's syndrome: an unusual association. Int J Urol. 2004;11(9):795-797.

5. Cowan DF. Quality assurance in anatomic pathology. An information system approach. Arch Pathol Lab Med. 1990;114(2):129-134.

6. Dworak O. Synoptic surgical pathology reporting. Hum Pathol. 1992;23(1):85-86.

7. Leslie KO, Rosai J. Standardization of the surgical pathology report: formats, templates, and synoptic reports. Semin Diagn Pathol. 1994;11(4): 253-257.

8. Markel SF, Hirsch SD. Synoptic surgical pathology reporting. Hum Pathol. 1991;22(8):807-810.
9. Nochomovitz LE. Application of synoptic reports. Arch Pathol Lab Med. 1998;122(6):493-494.

10. Whang M, Katz L, Ongcapin E, et al. Nephrogenic adenomas occurring in a patient with simultaneous kidney-pancreas transplant. Urology. 2000;55(6):949.

11. Piper NY, Thompson IM. Large nephrogenic adenoma following transurethral resection of the prostate. J Urol. 1999;161(2):605.

12. Mohanty SK, Piccoli AL, Devine LJ, et al. Synoptic tool for reporting of hematological and lymphoid neoplasms based on World Health Organization classification and College of American Pathologists checklist. BMC Cancer. 2007;7:144.

13. Tobias J, Chilukuri R, Komatsoulis GA, et al. The CAP cancer protocols - a case study of caCORE based data standards implementation to integrate with the Cancer Biomedical Informatics Grid. BMC Med Inform Decis Mak. 2006;6:25.

\section{Publish your work in this journal}

Open Access Bioinformatics is an international, peer-reviewed, open access journal publishing original research, reports, reviews and commentaries on all areas of bioinformatics. The manuscript management system is completely online and includes a very quick and fair

\section{Dovepress}

peer-review system. Visit http://www.dovepress.com/testimonials.php to read real quotes from published authors. 